\title{
Quantum Hall effect in carbon nanotubes and curved graphene strips
}

\author{
E. Perfetto, ${ }^{1,3}$ J. González, ${ }^{1}$ F. Guinea, ${ }^{2}$ S. Bellucci, ${ }^{3}$ and P. Onorato ${ }^{3,4}$ \\ ${ }^{1}$ Instituto de Estructura de la Materia, Consejo Superior de Investigaciones Científicas, Serrano 123, 28006 Madrid, Spain \\ ${ }^{2}$ Instituto de Ciencia de Materiales, Consejo Superior de Investigaciones Científicas, Cantoblanco, 28049 Madrid, Spain \\ ${ }^{3}$ INFN Laboratori Nazionali di Frascati, P.O. Box 13, 00044 Frascati, Italy \\ ${ }^{4}$ Department of Physics “A. Volta," University of Pavia, Via Bassi 6, I-27100 Pavia, Italy
}

(Received 30 January 2007; revised manuscript received 3 May 2007; published 24 September 2007)

\begin{abstract}
We develop a long-wavelength approximation in order to describe the low-energy states of carbon nanotubes in a transverse magnetic field. We show that in the limit where the square of the magnetic length $l=\sqrt{\hbar c / e B}$ is much larger than the $\mathrm{C}$-C distance times the nanotube radius $R$, the low-energy theory is given by the linear coupling of a two-component Dirac spinor to the corresponding vector potential. We investigate in this regime the evolution of the band structure of zigzag nanotubes for values of $R / l>1$, showing that for radius $R$ $\approx 20 \mathrm{~nm}$ a clear pattern of Landau levels starts to develop for magnetic field strength $B \gtrsim 10 \mathrm{~T}$. The levels tend to be fourfold degenerate, and we clarify the transition to the typical twofold degeneracy of graphene as the nanotube is unrolled to form a curved strip. We show that the dynamics of the Dirac fermions leads to states which are localized at the flanks of the nanotube and that carry chiral currents in the longitudinal direction. We discuss the possibility of observing the quantization of the Hall conductivity in thick carbon nanotubes, which should display steps at even multiples of $2 e^{2} / h$, with values doubled with respect to those in the odd-integer quantization of graphene.
\end{abstract}

DOI: $10.1103 /$ PhysRevB.76.125430

\section{INTRODUCTION}

Two-dimensional carbon compounds with $s p^{2}$ bonding have attracted much attention recently due to the experimental observation of a number of novel electronic properties. It has been possible to measure the transport properties of a single layer of graphite (so-called graphene), providing evidence that the quasiparticles have a conical dispersion around discrete Fermi points. ${ }^{1,2}$ Carbon nanotubes can be also considered as the result of wrapping up the graphene sheet, leading to systems with unconventional transport properties that reflect the strong electron correlation. ${ }^{3,4}$

The metallic carbon nanotubes and the graphene sheet have in common that their low-energy electronic dispersion is governed by a massless Dirac equation, around each of the two Fermi points of the undoped systems. ${ }^{5-7}$ The appearance of an additional pseudospin quantum number intrinsic to the Dirac spectrum has allowed us to understand, for instance, the degeneracy of the molecular orbitals in the fullerenes, ${ }^{8}$ the quantization rule of the Hall conductivity in graphene, ${ }^{9,10}$ or the properties of the polarizability in carbon nanotubes. ${ }^{11}$

In this paper, we investigate the effects of a transverse magnetic field on the transport properties of the carbon nanotubes, making use of the description of the electronic states in terms of Dirac fermion fields. The low-energy graphene band structure can be obtained by taking a continuum limit in which the momenta are much smaller than the inverse of the C-C distance $a .^{5-7}$ In the case of carbon nanotubes under transverse magnetic field, a sensible continuum limit also requires that the square of the magnetic length $l^{2}=\hbar c / e B$ is made much larger than $a$ times the nanotube radius, so that lattice effects can be disregarded. In that limit, we will obtain a simple field theory of Dirac spinors coupled to the magnetic field, allowing us to investigate the dependence of different features of the band structure on the topology of the space.
PACS number(s): 73.22.-f, 73.43. $-\mathrm{f}, 75.75 .+\mathrm{a}$

We will see that carbon nanotubes of sufficiently large radius may have a quantum Hall regime, with a quantized Hall conductivity $\sigma_{x y}$. In the case of graphene, it has been shown that $\sigma_{x y}$ has plateaus at odd multiples of $2 e^{2} / h,{ }^{1,2}$ as a consequence of the peculiar Dirac spectrum. ${ }^{9,10}$ We will find that the different topology of the carbon nanotubes leads instead to a quantization in even multiples of the quantity $2 e^{2} / h$, with steps in $\sigma_{x y}$ which are doubled with respect to those in graphene. We will also show how the transition to the odd-integer quantization of graphene takes place, as the nanotube is unrolled into a curved strip.

\section{CONTINUUM LIMIT OF CARBON NANOTUBES IN TRANSVERSE MAGNETIC FIELD}

In this section, we show how to take the continuum limit of carbon nanotubes when the relevant electronic excitations have a wavelength much larger than the C-C distance $a$. We illustrate this long-wavelength limit in the case of zigzag nanotubes, noting that the procedure works similarly for different helicities. The zigzag nanotubes have a unit cell with length $3 a$, containing four transverse arrays of carbon atoms at different longitudinal positions $x_{j}=0, a, 3 a / 2$, and $5 a / 2$, as shown in Fig. 1. This introduces a flavor index $j$ $=1, \ldots, 4$ labeling inequivalent atoms in the unit cell. It is convenient to introduce the Fourier transform of the electron operator $\Psi\left(x_{j}+3 m a, n\right)$ with respect to the position of the carbon atoms $n=1,2, \ldots, N$ in each transverse section,

$$
\Psi\left(x_{j}+3 m a, n\right) \sim \sum_{p} e^{i 2 \pi n p / N} \Psi_{j}(m, p),
$$

where $m \in Z$ runs over the different cells. The index $p$ labels then the different one-dimensional subbands, 


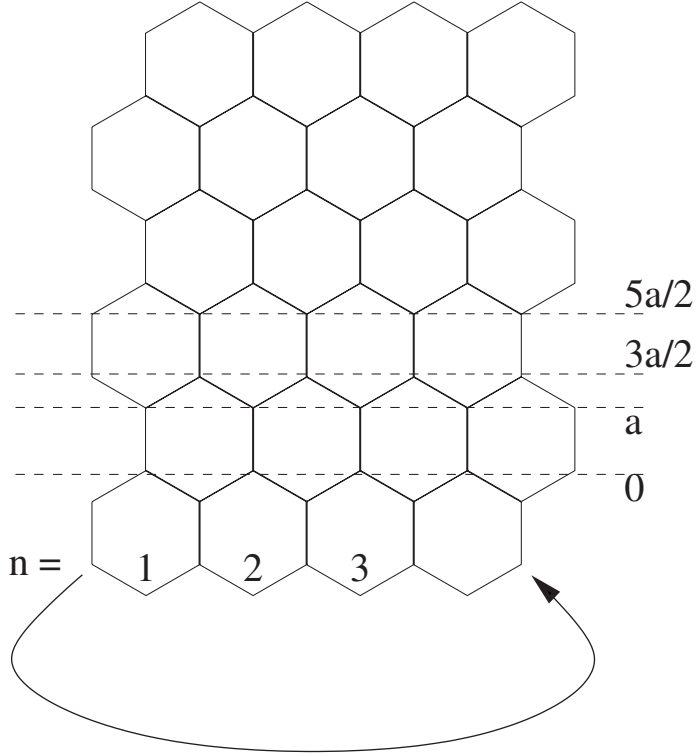

FIG. 1. Schematic representation of the lattice of a zigzag nanotube, showing the four different levels of inequivalent atoms in a unit cell.

$p=0, \pm 1, \ldots, \pm(N-1) / 2($ or $N / 2)$ for the case of odd (even) $N$.

Within the tight-binding approach, the Hamiltonian for the zigzag nanotube (without magnetic field) is given by

$$
\begin{aligned}
H_{T B}= & -t \sum_{p, m} \Psi_{1}^{+}(m, p) \Psi_{2}(m, p)-t \sum_{p, m} z_{p} \Psi_{2}^{+}(m, p) \Psi_{3}(m, p) \\
& -t \sum_{p, m} \Psi_{3}^{+}(m, p) \Psi_{4}(m, p)-t \sum_{p, m} z_{p}^{*} \Psi_{4}^{+}(m, p) \Psi_{1}(m+1, p) \\
& + \text { H.c. }
\end{aligned}
$$

where $t$ is the hopping integral and $z_{p} \equiv 1+\exp (i 2 \pi p / N)$. In the absence of magnetic field, the different subbands labeled by $p$ are decoupled and, after introducing the longitudinal momentum $k$, the Hamiltonian corresponds to a $4 \times 4$ matrix describing the unit cell:

$$
\left.\mathcal{H}_{p, p^{\prime}}\right|_{B=0}=-\delta_{p, p^{\prime}}\left(\begin{array}{cccc}
0 & 1 & 0 & e^{-i 3 k a} z_{p} \\
1 & 0 & z_{p} & 0 \\
0 & z_{p}^{*} & 0 & 1 \\
e^{i 3 k a} z_{p}^{*} & 0 & 1 & 0
\end{array}\right)
$$

The diagonalization of Eq. (3) leads, in general, to massive subbands with parabolic dispersion, with a gap $2 \Delta_{p}$ $=2 t|1-2 \cos (\pi p / N)|$. We note that whenever $N$ is a multiple of 3 , the gap vanishes for $p= \pm N / 3$ and we get two different couples of massless branches crossing in each case at $k=0$ but with opposite angular momentum around the nanotube. In general, the dispersive branches can be decoupled from the high-energy branches that appear near the top of the spectrum. It turns out that the low-energy dispersion corresponds to two reduced two-component spinors described each by the $2 \times 2$ Hamiltonian

$$
\left.\mathcal{H}_{p, p^{\prime}}\right|_{B=0}=\delta_{p, p^{\prime}}\left(\begin{array}{cc}
v_{F} \hbar k & \Delta_{p} \\
\Delta_{p} & -v_{F} \hbar k
\end{array}\right),
$$

where the Fermi velocity is $v_{F}=3 t a / 2 \hbar$. We see that the two components of each spinor, that we will denote by $\Psi_{R}$ and $\Psi_{L}$, correspond respectively to right- and left-moving modes, which are mixed by the massive (off-diagonal) term of the Hamiltonian.

The magnetic field is introduced with the usual prescription of correcting the transfer integral $t$ by appropriate phase factors,

$$
e^{i \phi}=\exp \left(i \frac{e}{\hbar c} \int_{\mathbf{r}}^{\mathbf{r}^{\prime}} \mathbf{A} \cdot \mathbf{d l}\right),
$$

depending on the vector potential A between nearestneighbor lattice sites at $\mathbf{r}$ and $\mathbf{r}^{\prime}$. For a magnetic field perpendicular to the carbon nanotube, the component normal to the surface has a periodic dependence on the angular variable $\theta$ around the tube. Taking the longitudinal distance $x$ and the angle $\theta$ as coordinates on the nanotube surface, a convenient choice of the gauge is given by

$$
\mathbf{A}=(R B \sin (\theta), 0),
$$

$R$ being the nanotube radius. The phase $\phi$ gets then a modulation around the nanotube, ${ }^{12}$

$$
\phi \propto a(e / \hbar c) B R \sin (2 \pi n / N), \quad n=1, \ldots, N .
$$

The feasibility of the continuum limit requires that $(e / \hbar c) B R a \ll 1$. When this condition is satisfied, we can deal with the linear approximation to the exponential (5). We observe then that the effect of the magnetic field is to mix a given subband $p$ with its nearest neighbors $p \pm 1$. This introduces another contribution to add to the Hamiltonian (3), given by

$\Delta \mathcal{H}_{p, p^{\prime}}=\delta_{p^{\prime}, p+1} t \frac{e B R a}{2 \hbar c}\left(\begin{array}{cccc}0 & 1 & 0 & -e^{-i 3 k a} z_{p+1} / 2 \\ -1 & 0 & z_{p} / 2 & 0 \\ 0 & -z_{p+1}^{*} / 2 & 0 & 1 \\ e^{i 3 k a} z_{p}^{*} / 2 & 0 & -1 & 0\end{array}\right)+\delta_{p^{\prime}, p-1} t \frac{e B R a}{2 \hbar c}\left(\begin{array}{cccc}0 & -1 & 0 & e^{-i 3 k a} z_{p-1} / 2 \\ 1 & 0 & -z_{p} / 2 & 0 \\ 0 & z_{p-1}^{*} / 2 & 0 & -1 \\ -e^{i 3 k a} z_{p}^{*} / 2 & 0 & 1 & 0\end{array}\right)$. 
By projecting again onto the two-dimensional low-energy space, $\Delta \mathcal{H}_{p, p^{\prime}}$ becomes

$$
\Delta \mathcal{H}_{p, p^{\prime}}=\delta_{p^{\prime}, p \pm 1}\left(\begin{array}{cc} 
\pm i v_{F}(e / c) B R / 2 & 0 \\
0 & \mp i v_{F}(e / c) B R / 2
\end{array}\right) .
$$

The Hamiltonian can be more easily expressed when acting on the two-component Dirac spinor,

$$
\Psi(k, \theta) \sim \sum_{p} e^{i \theta p} \Psi(k, p),
$$

depending on the angular variable $\theta$ around the tubule. We recall that there are, in fact, two different spinors describing states with opposite angular momentum around the nanotube. For a nanotube without gap, for instance, the Hamiltonian is in either case

$$
\mathcal{H}=\left(\begin{array}{cc}
v_{F} \hbar k+v_{F} \frac{e B R}{c} \sin (\theta) & -i\left(\hbar v_{F} / a\right) \partial_{\theta} \\
-i\left(\hbar v_{F} / a\right) \partial_{\theta} & -v_{F} \hbar k-v_{F} \frac{e B R}{c} \sin (\theta)
\end{array}\right),
$$

where the periodic modulation matches with the orientation of the magnetic field normal to the nanotube surface at $\theta$ $=0$. Expression (11) corresponds actually to the Dirac Hamiltonian with the usual prescription for the coupling to the vector potential, $\hbar k \rightarrow \hbar k+(e B R / c) \sin (\theta)$. We will see, however, that this simple gauge coupling does not hold in all cases, when discussing the curved graphene strips in the next section.

\section{LANDAU LEVEL QUANTIZATION}

We have diagonalized numerically the Hamiltonian $\mathcal{H}$ made of the sum of Eqs. (4) and (9), for different carbon nanotubes with radius $R \approx 20 \mathrm{~nm}$ and magnetic field $B$ varying between 0 and $20 \mathrm{~T}$. We have checked that for $a R / l^{2}$ $\ll 1$, the eigenstates of $\mathcal{H}$ provide a good approximation to the low-energy band structure obtained from the full tightbinding Hamiltonian incorporating the phase factors (5) for the different bonds. We present our results showing the evolution of the band structure computed from the Hamiltonian $\mathcal{H}$ for two different zigzag nanotubes, corresponding to $(510,0)$ and $(500,0)$ geometries in the usual notation. We observe that while the latter has a small gap in the absence of magnetic field, the evolution presented in Figs. 2 and 3 ends up in quite similar shapes for the band structure at strong magnetic field $(\approx 20 \mathrm{~T})$. The closure of the gap at a magnetic field $B<5 \mathrm{~T}$ is consistent with the results for semiconducting carbon nanotubes in Ref. 13. From there, we can infer that the magnetic field needed to close the gap of a nanotube with radius $R=10 \mathrm{~nm}$ must be of the order of $\sim 10 \mathrm{~T}$. This field strength would be reduced by a factor of 4 after doubling the nanotube radius, keeping the same ratio of $R / l$. For the larger radius $R \approx 20 \mathrm{~nm}$ in our analysis, we find that the band structures at $B=5 \mathrm{~T}$ in Figs. 2(b) and 3(b) only differ in the position of some unpaired subbands in the latter figure. At $B=10 \mathrm{~T}$, a discrepancy between Figs. 2(c) and 3(c) is
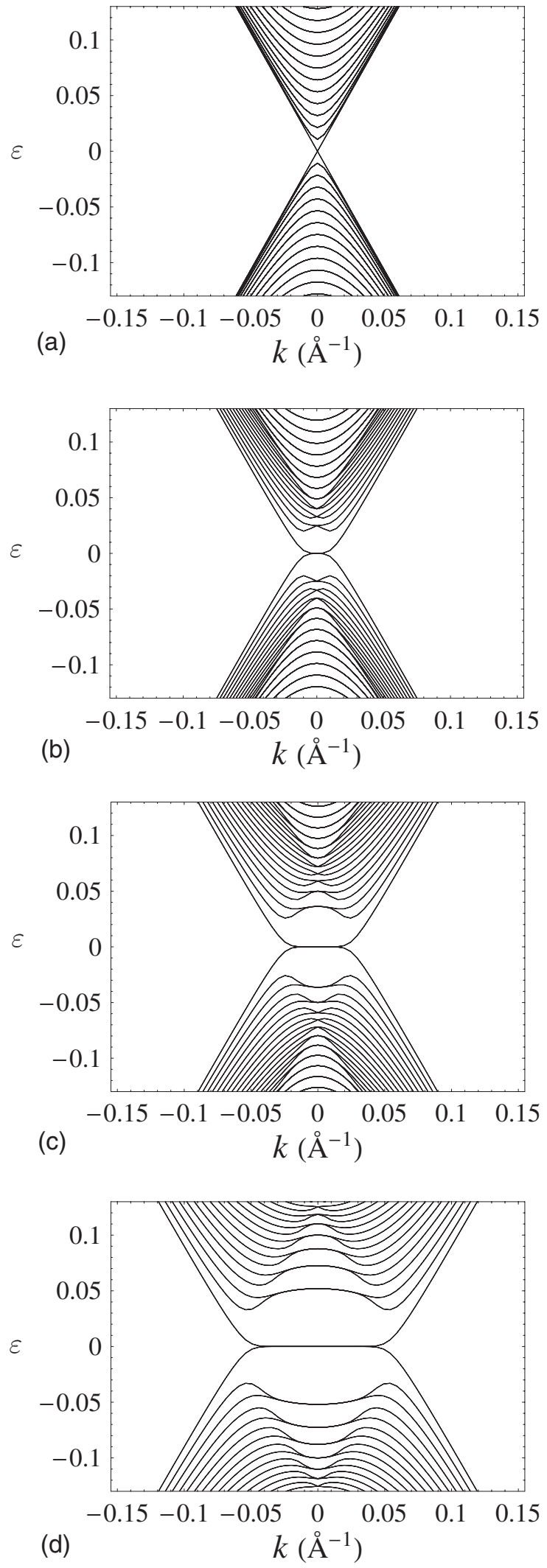

FIG. 2. Sequence of band structures of a zigzag $(510,0)$ nanotube with radius $R \approx 20 \mathrm{~nm}$ in transverse magnetic field for (a) $B$ $=0 \mathrm{~T}$, (b) $B=5 \mathrm{~T}$, (c) $B=10 \mathrm{~T}$, and (d) $B=20 \mathrm{~T}$. $B=20 \mathrm{~T}$ corresponds to $a R / l^{2} \approx 0.1$ and $R / l \approx 3.5$. Energy is in units of $t$ and momentum is in units of $\AA^{-1}$. 

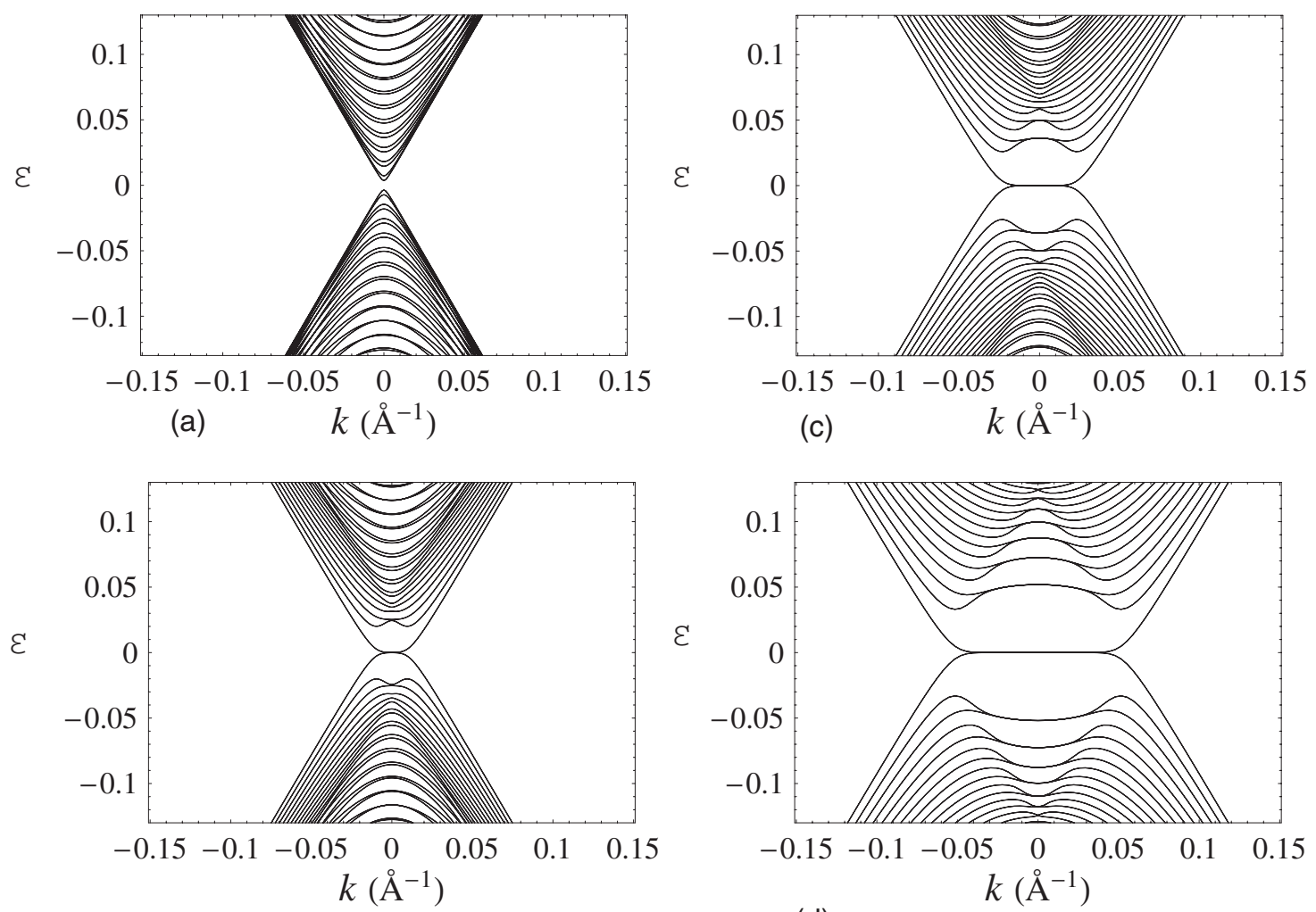

(b)

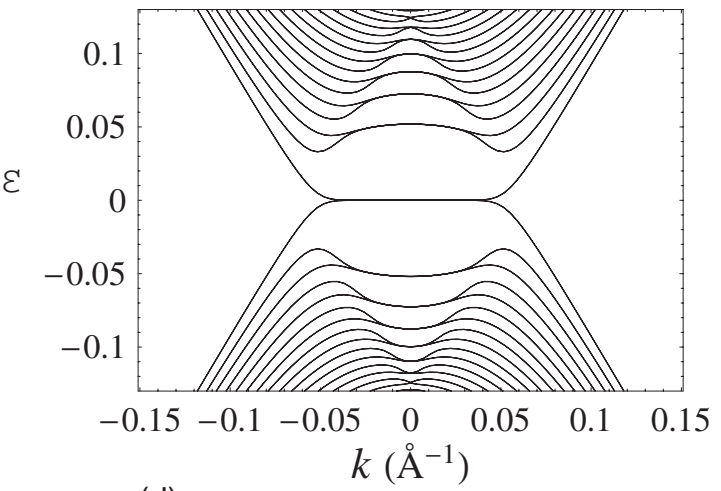

(d)

FIG. 3. Sequence of band structures of a zigzag $(500,0)$ nanotube with radius $R \approx 20 \mathrm{~nm}$ in transverse magnetic field for (a) $B=0 \mathrm{~T}$, (b) $B=5 \mathrm{~T}$, (c) $B=10 \mathrm{~T}$, and (d) $B=20 \mathrm{~T}$. Energy is in units of $t$ and momentum is in units of $\AA^{-1}$.

only found in the high-energy part of the plots, and at $B$ $=20 \mathrm{~T}$, the band structures for the metallic and the semiconducting nanotube presented in Figs. 2(d) and 3(d) are practically similar. This illustrates a more general result, which is that the form of the low-energy Landau subbands does not depend on the particular geometry of nanotubes with similar radius at such strong magnetic fields.

We have to point out, however, that the evolution of the band structure of the thick nanotubes considered here is quite different from that of carbon nanotubes with typical radius $(\sim 1 \mathrm{~nm})$ in strong magnetic fields. The latter have been investigated in Ref. 14, where typical oscillations have been reported in the low-energy levels of carbon nanotubes with $R \sim 1 \mathrm{~nm}$ as the magnetic field is increased to ratios of $R / l$ $=3$. The reason why the low-energy levels do not stabilize at increasing magnetic field can be traced back to the fact that for such thin carbon nanotubes, there is no regime where the continuum limit with $a R / l^{2} \ll 1$ can be realized. In these cases, by the time that we have $R \gtrsim l$, the magnetic length cannot be much larger than the $\mathrm{C}-\mathrm{C}$ distance, so that a quantum Hall regime cannot exist in thin carbon nanotubes of typical radius. This can also be appreciated in the results of Ref. 15, where the density of states of several carbon nanotubes is presented at very large magnetic fields, with a marked difference between the cases of thin and thick nanotubes. It has been shown, for instance, that the density of states for nanotube radius $R \approx 14 \mathrm{~nm}$ already resembles that of the parent graphene system, with clear signatures of Landau subbands in the low-energy part of the spectrum.
As presented in Figs. 2 and 3, our thick nanotubes develop, in general, two valleys at zero energy (that appear superposed in the figures) expanding around the two Fermi points of the parent graphene system at $B=0$. We see that flat Landau levels start developing already at $B=10 \mathrm{~T}$ [Fig. $2(\mathrm{c})]$. The existence of a zero-energy level at $k=0$ has been shown to be a robust property of carbon nanotubes in a transverse magnetic field. ${ }^{16,17}$ We have checked that for large magnetic field strength [as in Fig. 2(d)], the energy levels at $k=0$ follow the quantization rule $\varepsilon_{n} \propto \sqrt{n},{ }^{17}$ which is peculiar of graphene. ${ }^{12,18}$ The point that we want to stress here is our observation that the levels at $k=0$ are fourfold degenerate, including the zero-energy level, for any kind of nanotube geometry. This is in contrast with the case of planar graphene, where the zero-energy level is doubly degenerate. The reduction in the number of zero modes comes from the fact that the boundary conditions in the plane impose, in general, the hybridization of states with opposite transverse momentum, while they are otherwise independent in the tubular geometry. As we will see, this bears a direct relation to the quantization of the Hall conductivity in even multiples of $2 e^{2} / h$ in the carbon nanotubes.

A relevant question is how the fourfold degeneracy of the above band structure can evolve into a picture consistent with the odd-integer quantization of the Hall conductivity in the planar graphene samples. This can be clarified by studying the change in the band structure after cutting the nanotube along the longitudinal direction. If we cut along a maximum in the normal component of the magnetic field, this 


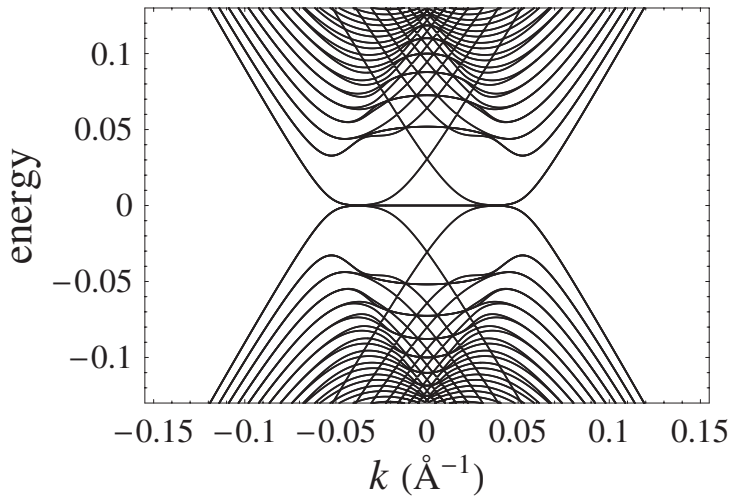

FIG. 4. Band structure corresponding to a zigzag nanotube with a cut in the longitudinal direction, for $R \approx 20 \mathrm{~nm}$ and $B=20 \mathrm{~T}$ (in the same units as in Fig. 2).

leads to a strip with a dependence of the tight-binding phase as in Eq. (7)

$$
\phi \propto a(e / \hbar c) B R \sin (2 \pi n / N) .
$$

We may obtain the band structure from the spectrum of an imaginary nanotube made by matching two copies of the strip, after projection onto the set of odd eigenmodes of the nanotube (which correspond to the stationary waves in the transverse direction of the strip ${ }^{19}$ ). The phase $\phi$ in the imaginary nanotube has to keep the mirror symmetry between the two copies. This leads to a function which does not have continuous derivative at the matching point of the two strips, with an infinite expansion in the modes around the nanotube. The corresponding interaction term is

$$
\begin{aligned}
\Delta \mathcal{H}_{p, p^{\prime}}= & \sum_{m} \delta_{p^{\prime}, p \pm 2 m+1} f\left(p-p^{\prime}\right) \\
& \times\left(\begin{array}{cc}
v_{F}(e / c) B R / 2 & 0 \\
0 & -v_{F}(e / c) B R / 2
\end{array}\right),
\end{aligned}
$$

with $f(p)=-8 / \pi\left(p^{2}-4\right)$. The band structure obtained after projection of the spectrum of $\mathcal{H}$ onto the odd eigenmodes of the nanotube is presented in Fig. 4. We observe that states that were at zero energy in the plot of Fig. 2(d) form two subbands dispersing toward higher energies and two other dispersing downward. In these conditions, only one valley is left at zero energy, evidencing the transition to the oddinteger quantization of the Hall conductivity of graphene.

A more complete picture of this transition can be obtained by studying an intermediate situation, where the nanotube has been cut longitudinally as before and opened by an angle of $\pi$ in the transverse magnetic field. This case is simple to analyze since it corresponds to a strip with a modulation of the tight-binding phase given by

$$
\phi \propto a(e / \hbar c) B R \cos (\pi n / N) .
$$

We may form a nanotube by matching two copies of the strip, obtaining again the band structure of the latter by projection of the spectrum of the nanotube onto eigenmodes with odd angular dependence. Now, the doubling of Eq. (14) realizing the mirror symmetry between the two copies gives back the same function $\phi$, and the interaction term becomes

$$
\Delta \mathcal{H}_{p, p^{\prime}}=\delta_{p^{\prime}, p \pm 1}\left(\begin{array}{cc}
v_{F}(e / c) B R / 2 & 0 \\
0 & -v_{F}(e / c) B R / 2
\end{array}\right) .
$$

The band structure obtained from the diagonalization of the full Hamiltonian $\mathcal{H}$ has a shape similar to that shown in Fig. 2 . The important difference is that now the projection onto the odd eigenmodes of the nanotube reduces by a factor of 2 the number of states, leaving all the subbands nondegenerate. We find therefore that in the curved strip which is formed by opening the nanotube by an angle of $\pi$, the zero-energy level is already twofold degenerate, while the rest of subbands coalesce in pairs at low momentum into twofold degenerated levels. This is actually the kind of degeneracy that leads to the odd-integer quantization of the Hall conductivity in the planar graphene samples. ${ }^{9,10}$

From the above results, we may also infer that the band structure of the carbon nanotube can be obtained from the superposition of the subbands of two nanotube halves in the transverse magnetic field. We can think of these two curved strips as the upper and the lower half of the carbon nanotube, leading to a picture in which gluing or not the two strips should be immaterial for the purpose of reproducing the nanotube band structure. It can be shown that this is actually the case, by looking at the result of cutting the nanotube at one of the flanks, where the normal component of the magnetic field vanishes. Unrolling then the nanotube leads to a strip with a modulation of the tight-binding phase,

$$
\phi \propto a(e / \hbar c) B R \cos (2 \pi n / N) .
$$

Its band structure can be deduced again from that of an imaginary nanotube made by matching two copies of the strip, with the same phase $\phi$, after projection onto the set of odd eigenmodes of the nanotube. This nanotube model has an interaction term

$$
\Delta \mathcal{H}_{p, p^{\prime}}=\delta_{p^{\prime}, p \pm 2}\left(\begin{array}{cc}
v_{F}(e / c) B R / 2 & 0 \\
0 & -v_{F}(e / c) B R / 2
\end{array}\right) .
$$

The full Hamiltonian $\mathcal{H}$ has now an even dependence on the quantum number $p$, and it can be checked that the result of projecting the spectrum onto the eigenmodes with odd angular dependence produces, in fact, a band structure which is

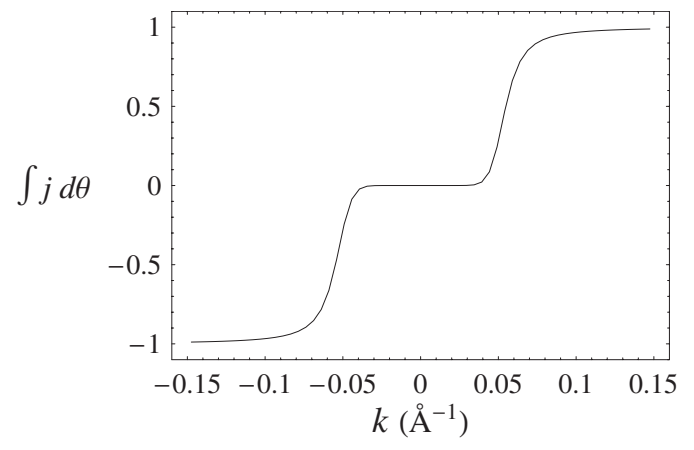

FIG. 5. Plot of the integral of the current $j$ over the angular variable $\theta$ (in units of $v_{F}$ ) for states in the lowest Landau subband of Fig. 2(d). 

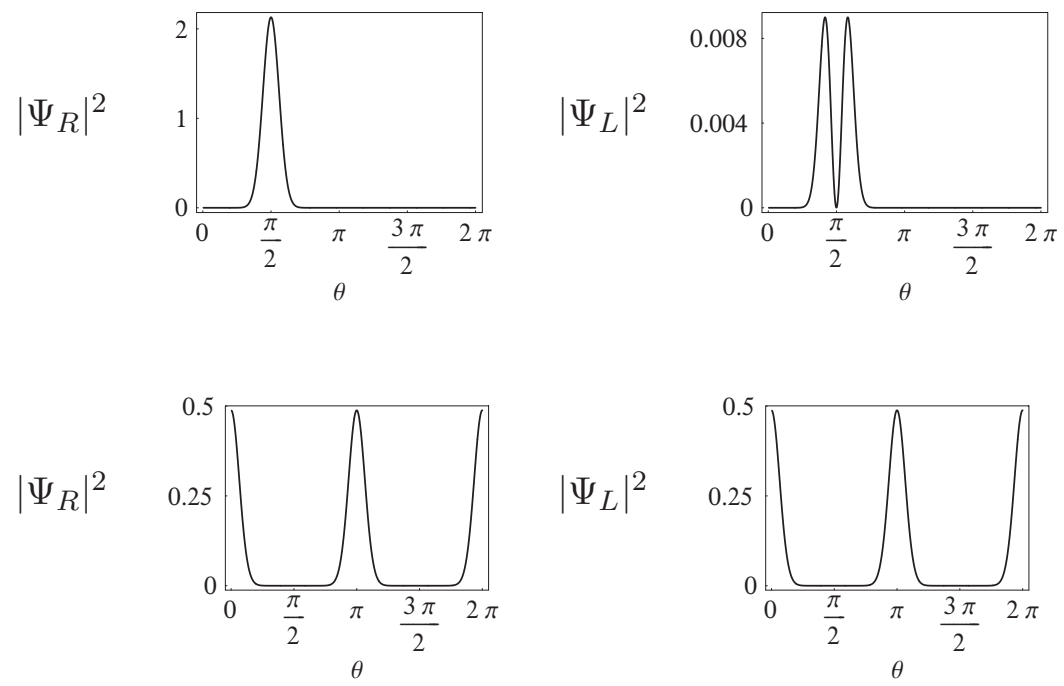

FIG. 6. Angular distribution of the eigenfunctions in the lowest Landau subband of Fig. 2(d). The panels correspond, from top to bottom, to longitudinal momenta $k=0.15,0,-0.15$ (in units of $\AA^{-1}$ ).
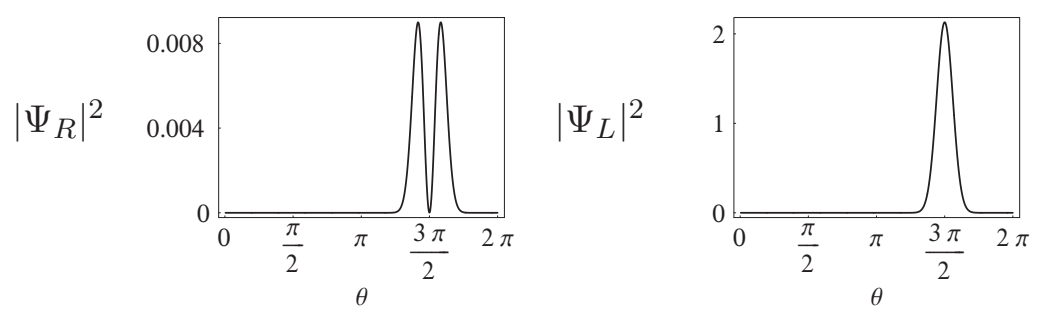

completely similar to that in Fig. 2. This provides the final evidence that the doubling of the spectrum of the carbon nanotube can be understood precisely from the doubling of the geometry in two equivalent upper and lower nanotube halves, which contribute equally to the spectrum in the transverse magnetic field.

\section{CURRENT QUANTIZATION}

We next clarify the way in which the dispersive branches of the band structure are connected to the quantization of the Hall conductivity in the carbon nanotubes. The outer dispersive branches correspond to states which are localized at the flanks of the nanotube. ${ }^{16}$ This suggests the possibility that, despite having no boundary, the carbon nanotubes may support edge states in a similar fashion as in systems with planar geometry. It can be shown actually that the current carried in the longitudinal direction by the states in the outer dispersive branches is quantized. In this context, the definition of the current $j$ must be consistent with the dynamics governed by the Hamiltonian (11). In real space, the equation of motion for the Dirac spinor $\Psi(x, \theta)$ reads

$$
\begin{aligned}
i \hbar \partial_{t}\left(\begin{array}{c}
\Psi_{R} \\
\Psi_{L}
\end{array}\right) & \\
= & \left(\begin{array}{cc}
-i v_{F} \hbar \partial_{x}+v_{F} \frac{e B R}{c} \sin (\theta) & -i\left(\hbar v_{F} / a\right) \partial_{\theta} \\
-i\left(\hbar v_{F} / a\right) \partial_{\theta} & i v_{F} \hbar \partial_{x}-v_{F} \frac{e B R}{c} \sin (\theta)
\end{array}\right) \\
& \times\left(\begin{array}{c}
\Psi_{R} \\
\Psi_{L}
\end{array}\right) .
\end{aligned}
$$

From Eq. (18), we derive the continuity equation

$$
\partial_{t}\left(\Psi_{R}^{+} \Psi_{R}+\Psi_{L}^{+} \Psi_{L}\right)=-v_{F} \partial_{x}\left(\Psi_{R}^{+} \Psi_{R}-\Psi_{L}^{+} \Psi_{L}\right),
$$

which dictates the expression of the current

$$
j=v_{F}\left(\Psi_{R}^{+} \Psi_{R}-\Psi_{L}^{+} \Psi_{L}\right) .
$$

The result of computing the integral over $\theta$ of the current $j$ for states in the lowest-energy subbands is presented in Fig. 5. It turns out that, in general, the states corresponding to the flat part of the Landau level do not carry any current in the longitudinal direction, while the states in the dispersive branches saturate quickly the maximum value $v_{F}$ as the dispersion approaches a constant slope.

Regarding the spatial distribution, there is also a clear correspondence between the localization of the states in the angular variable $\theta$ and the value of the current. This can be appreciated from inspection of the eigenstates of the Hamiltonian (11). We have presented in Fig. 6 the angular distribution of states from the lowest Landau subband for $B$ $=20 \mathrm{~T}$. Each eigenfunction is, in general, localized around a certain value of the angular variable $\theta$. We observe, for instance, that the states at $k=0$ have Gaussian wave functions localized at $\theta=0$ or $\theta=\pi$, with the contribution to the current from the left component compensating exactly that from the right component. For positive (negative) longitudinal momentum, the states in the flat zero-energy level are localized at angles between 0 and $\pi / 2(3 \pi / 2)$ or between $\pi$ and $\pi / 2$ $(3 \pi / 2)$, depending on the subband chosen. For the states in the dispersive branches, the eigenfunctions are centered around $\pi / 2$ (for a right branch) or $3 \pi / 2$ (for a left branch). Here, the role of the magnetic field is to separate left-moving 
and right-moving currents at the opposite sides of the tube. We actually observe that there is a large mismatch between $\left|\Psi_{L}\right|$ and $\left|\Psi_{R}\right|$ for states in the dispersive branches, which turn out to carry nonvanishing chiral currents flowing at the flanks of the nanotube.

The localization of the current in the states of the dispersive branches opens the possibility of observing the quantization of the Hall conductivity in thick carbon nanotubes. We first remark that, in general, the value of the current $j$ (more precisely the integral over $\theta$ ) is proportional to the slope of the dispersion $\varepsilon(k)$ in the given subband. This can be seen by taking the derivative with respect to $k$ of the expectation value of Eq. (11) in the energy eigenstates, after use of the Hellmann-Feynman theorem:

$$
\frac{1}{\hbar} \frac{\partial}{\partial k}\langle\mathcal{H}\rangle=\frac{1}{\hbar}\left\langle\frac{\partial}{\partial k} \mathcal{H}\right\rangle=v_{F} \int d \theta\left(\Psi_{R}^{+} \Psi_{R}-\Psi_{L}^{+} \Psi_{L}\right) .
$$

Thus, we obtain

$$
\frac{1}{\hbar} \frac{\partial \varepsilon(k)}{\partial k}=\int d \theta j
$$

Let us consider first the case in which the Fermi level is above the zero-energy level but without crossing the next Landau subband. If we denote by $\varepsilon_{0}(k)$ the dispersion of the lowest Landau subband, we have from Eq. (22) that the total longitudinal current $I$ is given then by the sum of $(e / \hbar) \partial \varepsilon_{0}(k) / \partial k$ over all the filled modes in the energy range between the respective chemical potentials $\varepsilon_{L}$ and $\varepsilon_{R}$ at the two nanotube flanks, ${ }^{20}$

$$
I=\frac{e}{\hbar} \int_{\text {filled states }} \frac{d k}{2 \pi} \frac{\partial \varepsilon_{0}(k)}{\partial k} .
$$

Provided that the Hall probes used to measure the difference in the chemical potential are noninvasive, as proposed below, the Hall voltage $V_{H}$ measured by the probes has to be given by $\left(\varepsilon_{R}-\varepsilon_{L}\right) / e .^{21}$ Therefore, the Hall conductivity, defined by $\sigma_{x y}=I / V_{H}$, must have a first plateau as a function of the filling level, with a quantized value given by the spin degeneracy and the doubling of the subbands shown in Fig. 2(d):

$$
\sigma_{x y}=4 \frac{e^{2}}{h}
$$

As the filling level is increased, the situation changes when the Fermi level starts crossing the bumps with parabolic dispersion shown in Fig. 2(d). The current given by Eq. (20) is again proportional to the slope of the energy dispersion. Thus, when the Fermi level intersects a parabolic branch, there are two opposite contributions to the longitudinal current $I$ from the respective Fermi points. As the filling level is further increased, however, the Fermi points move far apart, and the contributions to the current $I$ depend on the profile of the Hall potential across the section of the nanotube.

When the Fermi level crosses several of the dispersive branches shown in Fig. 2(d), there is the question of how the total current $I$ is distributed among the different subbands. This problem has been addressed in the context of the quan-

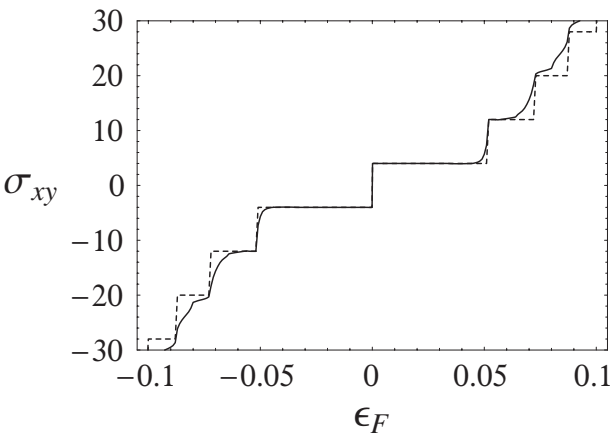

FIG. 7. Plot of the Hall conductivity $\sigma_{x y}$ (in units of $e^{2} / h$ ) as a function of the position of the Fermi level $\varepsilon_{F}$ in the band structure of Fig. 2(d). The full line corresponds to a linear Hall voltage drop and the dashed line to a sharp voltage drop in the bulk of the nanotube.

tum Hall effect in quantum wires, in experimental studies measuring the spatial profile of the Hall potential. ${ }^{22}$ It has been shown that such a spatial dependence is dictated by the compressibility of the electron liquid. In the case of the carbon nanotubes, the key point is the very limited screening afforded by such low-dimensional systems. It therefore seems reasonable to assume that the Hall potential drop is going to be approximately linear in the bulk of the nanotube. ${ }^{21}$ In these conditions, we have computed the Hall conductivity as a function of the filling level. For the inner Landau subbands in Fig. 2(d), the difference in the chemical potential between right and left branches $\varepsilon_{R}-\varepsilon_{L}$ is, in general, below the potential measured by the Hall contacts. The contribution of each inner dispersive branch to the Hall conductivity then turns out to be smaller than the quantized value from the outermost edge states. Consequently, an approximate quantization of $\sigma_{x y}$ is observed above the first plateau, as shown in Fig. 7, with steps according to the degeneracy of the subbands:

$$
\sigma_{x y} \approx(2+4 n) 2 \frac{e^{2}}{h} .
$$

The appearance of sharper steps in the Hall conductivity is achieved, in general, in samples with larger transverse size, and it can be also favored by the use of wider Hall contacts that induce a smoother drop of the Hall voltage at the edges. ${ }^{21}$ In practice, a real measure of the Hall conductivity may then be between the two curves displayed in Fig. 7.

\section{CONCLUSION}

We have shown that for thick carbon nanotubes in a transverse magnetic field, the transport properties are governed by the states localized at the flanks of the nanotube, which carry quantized currents in the longitudinal direction.

For nanotubes with a radius $R \approx 20 \mathrm{~nm}$, in a magnetic field of $\approx 20 \mathrm{~T}$, the band structure already shows a clear pattern of Landau levels. This opens the possibility of observing the quantization of the Hall conductivity in multi- 
walled nanotubes, where typically only the outermost shell is contacted by electrodes in transport experiments. In a suitable experimental setup, a metallic gate should be prepared as part of the substrate, to be used as a voltage probe contacting the bottom of the nanotube. The magnetic field should be oriented parallel to the substrate and perpendicular to the carbon nanotube. Then, by establishing a fixed current along the nanotube, changes in the Hall voltage at the top of the nanotube have to be observed upon variation of the magnetic field strength or the chemical potential in the nanotube. Longitudinal currents of the order of $\sim 1 \mu \mathrm{A}$ are quite affordable and, according to Eq. (25), they have to give rise to Hall voltages of the order of $\sim 1 \mathrm{mV}$. A scanning tunneling microscope (STM) tip may be used to contact the top part of the nanotube and to measure the voltage with respect to that of the metallic gate at the bottom. As the STM device may easily appreciate differences of the order of $\sim 10^{-4} \mathrm{eV}$, it has to be possible to observe at least the first steps in the Hall voltage implied by the quantization rule (25).

The observation of the plateaus in the Hall voltage should be fairly insensitive to the presence of moderate disorder in the nanotube samples, as long as the effect rests on the existence of chiral currents at opposite flanks of a nanotube. The overlap between states with currents flowing in opposite directions is exponentially small, so that the chiral currents cannot suffer significant backscattering from impurities or lattice defects. It is only at the electrodes, where the chiral currents meet, that backscattering may appear. As usually done in the context of the Hall effect in mesoscopic wires, this may be accounted for by means of a suitable transmission coefficient that would reflect as an additional factor in the relation between the longitudinal current and the Hall voltage. ${ }^{23}$

Finally, we remark that the absence of significant backscattering interactions leads to good perspectives to measure the properties of a robust chiral liquid at the flanks of the nanotube, which could be accomplished, in particular, by means of scanning tunneling spectroscopy.

\section{ACKNOWLEDGMENTS}

The financial support of the Ministerio de Educación y Ciencia (Spain) through Grants Nos. FIS2005-05478-C0201/02 and INFN05-14 is gratefully acknowledged. F.G. acknowledges funding from the European Union under Contract No. 12881 (NEST). S.B. and P.O. acknowledge the support of the grant 2006 PRIN "Sistemi Quantistici Macroscopici-Aspetti Fondamentali ed Applicazioni di strutture Josephson Non Convenzionali." E.P. was also supported by INFN under Grant No. 10068.
${ }^{1}$ K. S. Novoselov, A. K. Geim, S. V. Morozov, D. Jiang, M. I. Katsnelson, I. V. Grigorieva, S. V. Dubonos, and A. A. Firsov, Nature (London) 438, 197 (2005).

${ }^{2}$ Y. Zhang, Y.-W. Tan, H. L. Stormer, and P. Kim, Nature (London) 438, 201 (2005).

${ }^{3}$ R. Egger and A. O. Gogolin, Phys. Rev. Lett. 79, 5082 (1997).

${ }^{4}$ C. Kane, L. Balents, and M. P. A. Fisher, Phys. Rev. Lett. 79, 5086 (1997).

${ }^{5}$ D. P. DiVincenzo and E. J. Mele, Phys. Rev. B 29, 1685 (1984).

${ }^{6}$ J. González, F. Guinea, and M. A. H. Vozmediano, Nucl. Phys. B 406, 771 (1993).

${ }^{7}$ C. L. Kane and E. J. Mele, Phys. Rev. Lett. 78, 1932 (1997).

${ }^{8}$ J. González, F. Guinea, and M. A. H. Vozmediano, Phys. Rev. Lett. 69, 172 (1992).

${ }^{9}$ N. M. R. Peres, F. Guinea, and A. H. Castro Neto, Phys. Rev. B 73, 125411 (2006).

${ }^{10}$ V. P. Gusynin and S. G. Sharapov, Phys. Rev. Lett. 95, 146801 (2005).

${ }^{11}$ D. S. Novikov and L. S. Levitov, Phys. Rev. Lett. 96, 036402 (2006)
${ }^{12}$ R. Saito, G. Dresselhaus, and M. S. Dresselhaus, Physical Properties of Carbon Nanotubes (Imperial College Press, London, 1998), Chap. 6.

${ }^{13}$ J. P. Lu, Phys. Rev. Lett. 74, 1123 (1995).

${ }^{14}$ R. Saito, G. Dresselhaus, and M. S. Dresselhaus, Phys. Rev. B 53, 10408 (1996).

${ }^{15}$ N. Nemec and G. Cuniberti, Phys. Rev. B 74, 165411 (2006).

${ }^{16}$ H. Ajiki and T. Ando, J. Phys. Soc. Jpn. 62, 1255 (1993); 65, 505 (1996).

${ }^{17}$ H.-W. Lee and D. S. Novikov, Phys. Rev. B 68, 155402 (2003).

${ }^{18}$ J. W. McClure, Phys. Rev. 104, 666 (1956).

${ }^{19}$ L. Brey and H. A. Fertig, Phys. Rev. B 73, 235411 (2006).

${ }^{20} \mathrm{We}$ connect here with the standard derivation of the quantization of the Hall conductivity in a nonrelativistic system, as given by B. I. Halperin, Phys. Rev. B 25, 2185 (1982).

${ }^{21}$ H. Akera and T. Ando, Phys. Rev. B 39, 5508 (1989).

${ }^{22}$ E. Ahlswede, P. Weitz, J. Weis, K. von Klitzing, and K. Eberl, Physica B 298, 562 (2001).

${ }^{23}$ M. Büttiker, Phys. Rev. B 38, 9375 (1988). 